\title{
The S2 Baseband Processing System for Phase-coherent Pulsar Observations
}

\author{
R. Wietfeldt (ISTS), W. Van Straten, D. Del Rizzo, N. Bartel (York \\ University), W. Cannon (ISTS/York University), M. Bailes (Melbourne \\ University), J. Reynolds, W. Wilson (ATNF)
}

\begin{abstract}
The phase-coherent recording of pulsar data and subsequent software dispersion removal provide a flexible way to reach the limits of high time resolution, useful for more precise pulse timing and the study of fast signal fluctuations within a pulse. Because of the huge data rate and lack of adequate recording and computing capabilities, this technique has been used mostly only for small pulsar data sets. In recent years, however, the development of very capable, reasonably inexpensive high-speed recording systems and computers has made feasible the notion of pulsar baseband recording and subsequent processing with a workstation/computer. In this paper we discuss the development of a phase-coherent baseband processing system for radio pulsar observations. This system is based on the S2 VLBI recorder developed at ISTS/York University in Toronto, Canada. We present preliminary first results for data from the Vela pulsar, obtained at Parkes, Australia, and processed at ISTS/York University, and discuss plans for future developments.
\end{abstract}

\section{System Description}

The S2 baseband system is shown in schematic form in Figure 1. Developed originally for VLBI applications [1], the S2 recorder is based on the use of commercial VHS tape transports (VCR's), modified for use in digital high-density, high-data rate applications. The key features of the $\mathrm{S} 2$ baseband system are the $\mathrm{S} 2$ recorder, the efficient mechanism for high volume, high data rate transfer from tape to computer, and the tightly-coupled control interface to the computer which enables automated data transfer and processing. A single S2 'tapeset' of eight SVHS tapes provides up to $500 \mathrm{~GB}$ storage, and an unattended operating time of up to 8.5 hours at the maximum data rate of $128 \mathrm{Mb} / \mathrm{s}$ or $16 \mathrm{MB} / \mathrm{s}$, corresponding typically to $16 \mathrm{MHz}$ bandwidth in two circular polarizations at 2-bit quantization, which generates a data rate of up to $1 \mathrm{~GB} /$ minute. For more challenging applications, multiple S2's may be used. The best computers of the day may require processing times exceeding real-time by a factor of a few.

\section{First Results and Future Developments/Applications}

First results are shown in Figure 2, corresponding to S-band observations of the Vela pulsar (PSR B0833-45) at Parkes on day 273, 1995. No de-dispersion was 


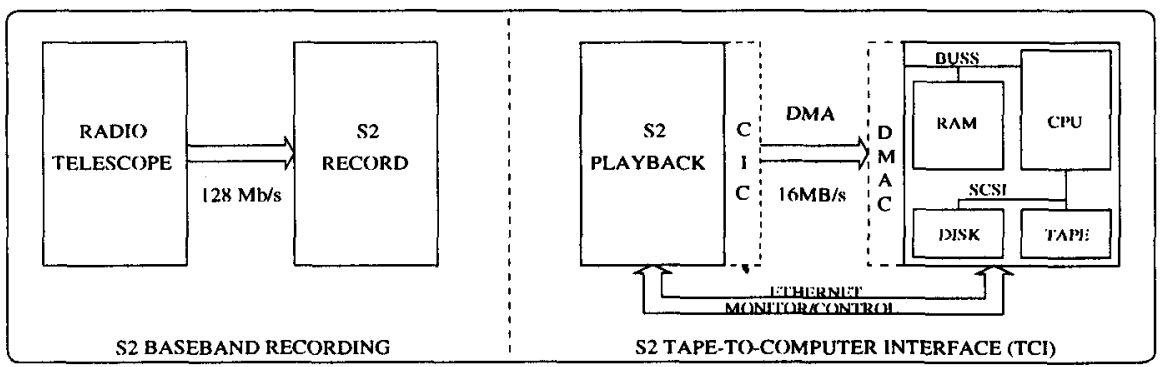

Figure 1. S2 Baseband Processing System Overview. Baseband digitized data are recorded on the $\mathrm{S} 2$ recorder at up to $128 \mathrm{Mb} / \mathrm{s}$. S2 tapes are shipped to the processing center, where data are transferred to computer via the S2 Tape-to-Computer Interface (TCI) at up to $16 \mathrm{MB} / \mathrm{s}$, with subsequent processing in software. Note: CIC ('Computer Interface Card') and DMAC ('DMA Card') are additional items to standard S2 and computer systems, respectively.

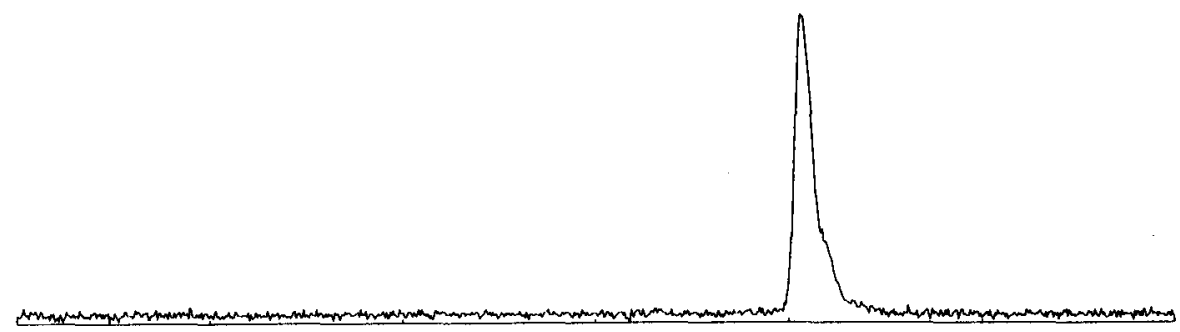

Figure 2. The average $60 \mathrm{~s}$ pulse profile of the Vela pulsar shown for a full period. The output of a $16 \mathrm{MHz}$ wide baseband channel was sampled at the Nyquist resolution of $31.25 \mathrm{~ns}$, converted to power, and then smoothed for display purposes to a time resolution of $100 \mu \mathrm{s}$.

attempted, but future plans for coherent de-dispersion will enable maximum time resolution. This baseband system has applications in many areas of pulsar research including pulsar timing, searches, single pulse studies, etc., and should also find applications in studies of transient sources such as flare stars. The reliability of the $\mathrm{S} 2$ will enable 'tight' embedded interaction with the controlling computer, necessary for successful automated, unattended processing.

\section{References}

[1] Wietfeldt et al., 'The S2 Recorder', Frontiers of VLBI, Universal Academy Press, Inc., Hirabayashi et al. (eds), 1991. 